\title{
Epigallocatechin-3-gallate in the prevention and treatment of hepatocellular carcinoma: experimental findings and translational perspectives
}

This article was published in the following Dove Medical Press journal:

Drug Design, Development and Therapy

\section{Sabrina Bimonte' \\ Vittorio Albino² \\ Mauro Piccirillo 2 \\ Aurelio Nasto ${ }^{3}$ \\ Carlo Molino ${ }^{4}$ \\ Raffaele Palaia ${ }^{2}$ \\ Marco Cascella'}

'Division of Anesthesia and Pain Medicine, Istituto Nazionale

Tumori, IRCCS - Fondazione G Pascale, Naples, Italy; ${ }^{2}$ Division of Hepatobiliary Surgical Oncology, Istituto Nazionale Tumori, IRCCS Fondazione G Pascale, Naples, Italy; ${ }^{3}$ UOC Chirurgia Generale ad Indirizzo Oncologico, POA Tortora, Pagani, Salerno, Italy; ${ }^{4} \mathrm{~A}$ Cardarelli Chirurgia Generale - AORN, Naples, Italy
Correspondence: Sabrina Bimonte Istituto Nazionale Tumori, IRCCS Fondazione G Pascale, 53 Via Mariano Semmola, Naples 80 I3I, Italy Email s.bimonte@istitutotumori.na.it

\begin{abstract}
Hepatocellular carcinoma (HCC), a primary liver malignancy, is one of the deadliest cancers worldwide. Despite orthotopic liver transplantation and hepatic resection representing the principal lines of treatment for this pathology, only a minority of patients can be resected owing to cirrhosis or late diagnosis. Keeping in mind the end goal of conquering these challenges, new alternative approaches have been proposed. Accumulating evidence has demonstrated that epigallocatechin-3-gallate (EGCG), the principal catechin of green tea with multiple biological properties, is able to modulate different molecular mechanisms underlying HCC, mainly through its antioxidant activity. In this article, we revise these findings reported in the literature, in order to highlight the potential roles of EGCG in the treatment of HCC. The CAMARADES criteria were applied for quality assessment of animal studies, and a narrative synthesis performed. New bits of information available for translational perspectives into clinical practice are addressed. Keywords: hepatocellular carcinoma, epigallocatechin-3-gallate, tumor progression, preclinical studies
\end{abstract}

\section{Introduction}

Hepatocellular carcinoma (HCC) is an aggressive liver cancer associated with a high rate of mortality worldwide. ${ }^{1,2}$ Orthotopic liver transplantation and surgical hepatic resection are considered the principal approaches for HCC treatment, but many patients are precluded from these therapies, due to unavailability of organs, cirrhosis, or late diagnosis. ${ }^{3}$ Systemic sorafenib-based therapy is an alternative choice for HCC patients in an advanced stage, although a significant number of patients are refractory to this multikinase-inhibitor $\mathrm{drug}^{4}$ and require further approaches, such as regorafenib, which is to date the only systemic treatment associated with survival improvement in HCC patients progressing on sorafenib treatment. ${ }^{5}$ Based on these premises, new alternative therapies for HCC treatment are much needed. Accumulating evidence has shown that the catechins and flavonoids contained in green tea (Camellia sinensis) confer to this plant cancer-chemopreventive effects. Particularly, epigallocatechin-3-gallate (EGCG), the most abundant active catechin found in green tea, induces apoptosis and inhibits tumor progression through its antioxidant and anti-inflammatory properties by modulating different signaling pathways in several types of cancer, including HCC (Figure 1). ${ }^{6-8}$ Also, it has been shown that EGCG behaves as a prooxidant in the presence of elevated concentrations of transition metals, mainly copper, thus leading to cytotoxic effects in cancer cells through the generation of reactive oxygen species (ROS), which are responsible for DNA breakage. ${ }^{9-12}$ submit your manuscript Dovepress f http in $\square$ 


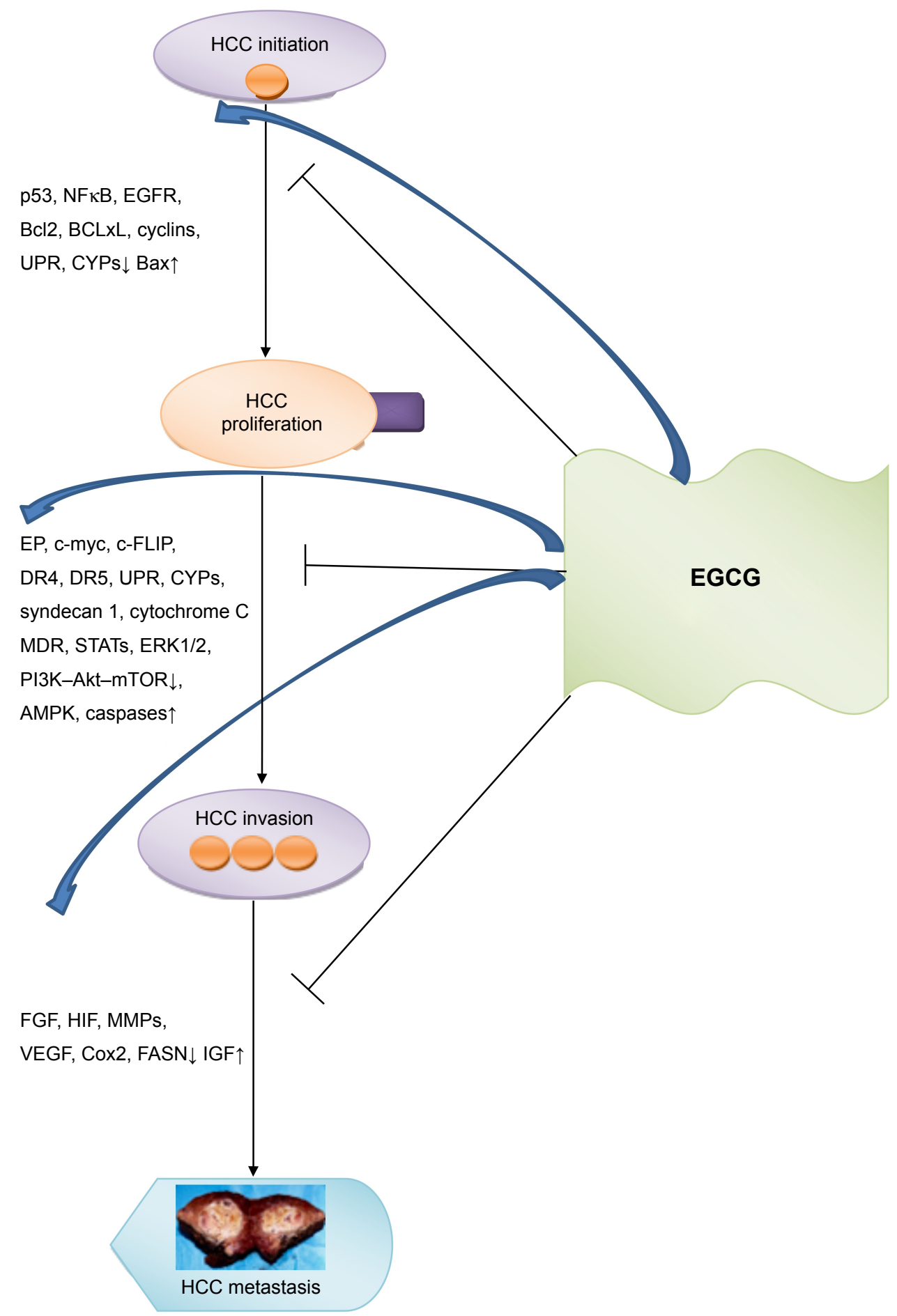

Figure I Principal targets modulated by EGCG in HCC progression.

Abbreviations: EGCG, epigallocatechin-3-gallate; HCC, hepatocellular cancer; UPR, unfolded protein response.

In this article, we revise these findings reported in the literature in order to highlight the potential roles of EGCG in the prevention and treatment of HCC and to offer a new contribution to conceivable applications into clinical practice. A narrative synthesis is also done for all studies considered, and the level of evidence for each included animal study was assessed by using a modified CAMARADES eleven-item checklist. ${ }^{13}$

\section{Chemistry of EGCG}

EGCG is the most abundant active catechin found in greentea extract, accounting for $65 \%$ of its content. Due to the presence of three heterocyclic rings into EGCG's chemical structure, as we previously reported, ${ }^{14}$ electron delocalization is favored, leading to quenching of free radicals. EGCG, like other tea catechins, has redox properties and reacts mainly 
with ROS. Particularly, the ability of EGCG to chelate free metal ions leads to prevention of ROS formation and the oxidation of catechins. ${ }^{9,15,16}$ Basically, its chemical structure not only confers antioxidant activities to EGCG but also favors its air oxidation, mainly in the presence of ions, leading to the formation of unstable catechin dimers. Moreover, as a consequence of the catechin structure, EGCG and other catechins are susceptible to several biotransformations, such as methylation, glucuronidation, and degradation, mediated by specific enzymes, mainly in the liver and in the small intestine. ${ }^{17-19}$ Finally, the hydrogen bonding capacity associated with EGCG's polyphenolic structures is responsible for reduced EGCG absorption. ${ }^{20}$

\section{Bioavailability of EGCG: constraints and upgrades}

Despite its health-promoting properties, EGCG is characterized by poor bioavailability, mainly due to its elevated instability, lower capacity to permeate the gastrointestinal tract under specific $\mathrm{pH}$ conditions, and active efflux. ${ }^{21-25}$ Therefore, its efficacy and application in clinical practice are very constrained and should be moved forward. For this purpose, several clinical trials have been conducted by controlling pharmacokinetic parameters, and have been reviewed by Mereles and Hunstein, ${ }^{26}$ in order to select the most appropriate dose and route of administration for EGCG intervention. Results from these studies suggest that adjustments in different parameters (eg, temperature and humidity $)^{27}$ and improvements in dietary habits (eg, not drinking hard water; EGCG intake on an empty stomach; dietary introduction of vitamins, $\omega_{3}$ polyunsaturated fatty acids, and piperine $)^{28-31}$ could enhance EGCG bioavailability. Additionally, the application of a nanodelivery system to encapsulate EGCG into specific nanoparticles (eg, chitosan and peptides) $)^{32,33}$ successfully enhance EGCG intestinal absorption, thus improving its bioavailability. In addition, a comparative impact has been likewise depicted by the engineering and blend of EGCG derivatives. ${ }^{34,35}$

\section{Preclinical assessments of EGCG anticancer effects in hepatocellular carcinoma}

\section{In vitro studies on hepatic cancer cell lines}

Accumulating preclinical evidence has shown that EGCG inhibits the growth and induces apoptosis of different liver cancer cells by acting via different molecular mechanisms (Table 1 and Figure 1).
The first report investigating the role of EGCG in hepatoma was from Nishida et al. ${ }^{36}$ The authors showed that EGCG was able to inhibit the growth and secretion of AFP in human hepatoma-derived PLC/PRF/5 cells without affecting their viability. Also, EGCG inhibited the growth of spontaneous hepatoma in $\mathrm{C} 3 \mathrm{H} / \mathrm{HeNCrj}$ mice without signs of toxicity. Subsequently, the inhibitory effects of EGCG on the invasion of hepatoma cells were proved in AH109A cells through the ROS-scavenging activity of EGCG. ${ }^{37}$ Uesato et al ${ }^{38}$ proved for the first time that EGCG inhibited the growth of HCT116 colorectal and HepG2 HCC cells, although with a lower effect with respect to (-)-epicatechin. A different molecular pathway underlying the effect of EGCG on apoptosis in HCC cells was described by Park et al. ${ }^{39}$ The authors demonstrated that EGCG diminished hypoxia-incited apoptosis in HepG2 cells and enhanced cell survival. These data support the hypothesis that EGCG could be considered a useful agent for HCC treatment. In fascinating in vitro and in vivo investigations, Nishikawa et $\mathrm{l}^{40}$ demonstrated that EGCG was able to induce apoptosis in HLE cells by inactivation of NF $\kappa B$ alone and in combination with TRAIL. Using in vitro approaches, Zhang et $\mathrm{al}^{41}$ showed that EGCG induced apoptosis of HCCLM6 cells by decreasing the mitochondrial membrane potential and by promoting $\mathrm{G}_{0} / \mathrm{G}_{1}$-phase cell-cycle arrest.

In addition to the apoptotic effects, other mechanisms, including the antiangiogenic effect, have clarified the potential role of EGCG on hepatocarcinoma tumor growth. Zhang et $\mathrm{al}^{42}$ showed that green-tea extract and EGCG significantly inhibited the accumulation of HIF $1 \alpha$ and its downstream target - VEGF expression - in both human cervical carcinoma (HeLa) and HepG2 cells. Interestingly, they proved that these results were obtained without affecting HIF $1 \alpha$ mRNA levels and by acting on PI3K/Akt and/ or ERK1/2 molecular mechanisms. In a study conducted on human HCC cells, SK-Hep1 and Pinus densiflora leaf extract and its phytochemicals, EGCG, EGC, and catechin gallate inhibited cell invasion and metastasis formation by altering the activities of MMPs, particularly gelatinase A (MMP2) and gelatinase B (MMP9), in a dose-dependent manner, ${ }^{43}$ and EGCG exhibited higher efficacy. Subsequently, Lee et $\mathrm{al}^{44}$ confirmed the ability of EGCG to suppress the cytotoxic effect induced by ethanol at lethal doses in HepG2 cells. In another study, an inhibitory role of EGCG on various types of the tyrosine-kinase receptor IGF1R in HepG2 cells was highlighted. ${ }^{45}$ Later on, Chen et al showed that EGCG was able to induce apoptosis in HepG2 and SMMC7721 cells by affecting the expression profiles of Cox 2 and BCL 2 and by activating caspase 9 and caspase $3 .{ }^{46}$ A potential role of EGCG in HCC treatment was suggested by Kaufmann et al 
Table I A summary of in vitro studies on the role of EGCG in hepatic cancer cell growth

\begin{tabular}{|c|c|c|c|}
\hline Cell line & Dose of EGCG & Molecular target(s) & Reference \\
\hline AHI09A & $10 \mathrm{mM}, 50 \mathrm{mM}$ & $\operatorname{ROS} \downarrow$ & 37 \\
\hline HCTII6, HepG2 & $\begin{array}{l}7.6 \pm 0.4 \mu \mathrm{M} \text { in HCTI I6, II } 2 \pm 0.5 \mu \mathrm{M} \\
\text { in HepG2 }\end{array}$ & - & 38 \\
\hline HepG2 & $12.5,25,50,100 \mu \mathrm{M}$ & $\begin{array}{l}\text { BCL2LI, CDHI, TACSTDI, PTK2 } \uparrow, \text { BAX, } \\
\text { CASP3, HIFIA } \downarrow\end{array}$ & 39 \\
\hline HLE, HepG2, Huh7 PLC/PRF/5 & $10,25,50,100 \mu \mathrm{g} / \mathrm{mL}$ & $\begin{array}{l}\text { Caspases 8, 9, and } 3 \uparrow, B C L 2 \alpha, B C L x L \\
\text { NFKB } \downarrow\end{array}$ & 40 \\
\hline HCCLM6 & $5-100 \mu \mathrm{g} / \mathrm{mL}$ & $\begin{array}{l}\text { MMP2, MMP9, FUSE, FUBPI, HSPBI, } \\
\text { CH60 } \downarrow \text {, NPM } \uparrow\end{array}$ & 41 \\
\hline HeLa, HepG2 & & HIFI $\alpha$, VEGF, PI3K/Akt, ERKI/2, mTOR $\downarrow$ & 42 \\
\hline SK-Hepl & $2 \mathrm{mg} \mathrm{FW} / \mathrm{mL}, 20 \mu \mathrm{g} / \mathrm{mL}$ & MMP2, MMP9 $\downarrow$ & 43 \\
\hline HepG2 & $1.0 \mathrm{mg} / \mathrm{mL}$ & & 44 \\
\hline $\begin{array}{l}\text { Huh7, PLC/PRF/5, HLF, HLE, HepG2, } \\
\text { and Hep3B }\end{array}$ & 20 or $40 \mu \mathrm{g} / \mathrm{mL}$ & $\begin{array}{l}\text { IGFI, IGF2, p-IGFIR, p-GSK3 } \beta, \text { p-ERK, } \\
\text { p-Akt, and p-STAT3 } \downarrow \text {, IGFBP3E } \uparrow\end{array}$ & 45 \\
\hline HepG2, SMMC772I & $\begin{array}{l}6.25,12.5,25,50,100,200 \text {, and } \\
400 \mu g / \mathrm{mL}\end{array}$ & Cox2, BCL2 $\downarrow$, caspases 9 and $3 \uparrow$ & 46 \\
\hline $\begin{array}{l}\text { Hep3B, two primary cultures established } \\
\text { from surgically resected HCC }\end{array}$ & $0-10 \mu \mathrm{M}$ & PARI/PAR4, p42, p44, MAPK $\downarrow$ & 47 \\
\hline $\begin{array}{l}\text { HLF, PLC/PRF/5, HepG2, Huh7, HLE, } \\
\text { and Hep3B }\end{array}$ & $0-100 \mu \mathrm{g} / \mathrm{mL}$ & VEGFR2, p-VEGFR2 $\downarrow$ & 48 \\
\hline HepG2, Нер3B & $0-160 \mu \mathrm{M}$ & $\begin{array}{l}\text { AMPK } \uparrow, \text { mTOR, FASN, ACC } \downarrow, \text { p53 in } \\
\text { HepG } 2 \downarrow, \text { p53 in Hep3B } \uparrow\end{array}$ & 49 \\
\hline HepG2 & $80,120,160 \mu \mathrm{mol} / \mathrm{L}$ & Smad7 $\downarrow$ & 50 \\
\hline BEL7404, BEL7404/Dox & $70,140,285,560,1,120 \mu \mathrm{g} / \mathrm{mL}$ & Dox chemosensitivity and cytotoxicity $\uparrow$ & 51 \\
\hline SMMC772I, Нер3B & $0-5 \mu \mathrm{M}$ & CBRI $\downarrow$ & 52 \\
\hline Нер3В & $\begin{array}{l}5,10,25 \text {, and } 50 \mu \mathrm{mol} / \mathrm{L} \text { in presence } \\
\text { or in absence of } 5 \mathrm{FU}\end{array}$ & Cox2, Akt, pAkt $\downarrow$, phospho-ACC, AMPK $\uparrow$ & 53 \\
\hline HepG2, MHCC97L, MHCC-97H, L02 & $100 \mu g / m L$ & $\mathrm{PGE}_{2} \downarrow, \mathrm{EPI} \downarrow$ & 54 \\
\hline HCCLM6 & $\begin{array}{l}0,5,10,20,30,40,50,60,80, \text { or } \\
100 \mu g / \mathrm{mL}\end{array}$ & $\begin{array}{l}\text { MMP2, MMP9, FUBPI, HSPBI, CH6O } \downarrow \text {, } \\
\text { NPM } \uparrow\end{array}$ & 41 \\
\hline HUVECs, Huh7 & Methyl-EGCG & VEGF, VEGFR2, p42/44 MAPK $\downarrow$ & 55 \\
\hline BEL-7402, QGY-7703 & $0-350 \mu \mathrm{M}$ & p-STAT3, cyclin DI, BCLxL, c-Myc, VEGF $\downarrow$ & 56 \\
\hline HepG2, SMMC772I, SK-HepI & $0,40,80,120 \mu \mathrm{M}$ & $\mathrm{PI} 3 \mathrm{~K}, \mathrm{Akt}, \mathrm{NF \kappa B} \downarrow$ & 57 \\
\hline Нер3В & $0,10,20,40 \mu \mathrm{g} / \mathrm{mL}$ & Autophagic genes $\downarrow$ & 46 \\
\hline HepG2 & $10,50,100,200 \mu \mathrm{M}$ & MMP9, syndecan I, FGF2 $\downarrow$ & 8 \\
\hline HCCLM6, HL7702 & $\begin{array}{l}0,5,10,20,30,40,60,80 \text {, and } \\
100 \mu g / \mathrm{mL} \text { in } \mathrm{HL} 7702,0,5,10,20 \text {, } \\
40,60,80,100,140,180,220 \text {, and } \\
260 \mu \mathrm{g} / \mathrm{mL} \text { in HCCLM6 }\end{array}$ & $\mathrm{BCL} 2, \mathrm{NF \kappa B} \downarrow, \mathrm{BA} X, \mathrm{p} 53$, caspases 9 and $3 \uparrow$ & 58 \\
\hline HepG2, MHCC97H, Нер3B & $0.02-20 \mu \mathrm{g} / \mathrm{mL}$ & OPN $\downarrow$ & 59 \\
\hline $\begin{array}{l}\text { HepG2, HCTII6, HLF, Huh7, HepG2, } \\
\text { CSCs }\end{array}$ & $0-75 \mu \mathrm{mol}$ & $\begin{array}{l}\text { DI33, Nanog, ATP-binding cassette } \\
\text { transporter genes } \downarrow, A B C C 1, A B C G 2, \text { Nek2, } \\
\text { p-Akt in CSCs } \downarrow\end{array}$ & 60 \\
\hline $\begin{array}{l}\text { HCC-LM3, SMMC772I, Нер3B, HepG2, } \\
\text { Huh7, QSG770I, LO2 }\end{array}$ & $25,50,100 \mu \mathrm{M}$ & PFK $\downarrow$ & 61 \\
\hline HepG2 & $50 \mu \mathrm{M}$ & $\begin{array}{l}\text { Dox-induced overexpression of PgP, } \\
\text { PI3K/Akt, Mek/ERK } \downarrow\end{array}$ & 62 \\
\hline HepG2 & $0-175 \mu \mathrm{M}$ & AFP $\downarrow$ & 63 \\
\hline
\end{tabular}

Abbreviations: CSCs, cancer stem cells; Dox, doxorubicin; EGCG, epigallocatechin-3-gallate; HCC, hepatocellular carcinoma; HUVECs, human umbilical vein endothelial cells; ROS, reactive oxygen species. 
in in vitro studies on the permanent liver carcinoma cell line HEP3B and two primary cultures surgically resected from HCC patients. Data from these studies showed that EGCG had an inhibitory effect on PAR1 and PAR4 (involved in HCC progression) and on p42/p44 MAPKs. ${ }^{47}$ Shirakami et $\mathrm{a}^{48}$ demonstrated an inhibitory role of EGCG on HCC progression by altering the activities of the tyrosine-kinase receptor VEGFR, which is involved in the angiogenesis of HCC. In vitro experiments were conducted on six human HCC cells - HLF, PLC/PRF/5, HepG2, Huh7, HLE, and Hep3B - and the major EGCG inhibitory effect on HCCcell growth was observed in Huh7 cells. This result was also confirmed by an in vivo nude mouse xenograft model generated by Huh7 cells injected subcutaneously in mice. Taken together, these results suggest that EGCG can be considered a promising agent for HCC treatment.

A chemopreventive and antilipogenic role of EGCG for HCC was highlighted by Huang et al. ${ }^{49}$ Using HepG2 (p53-positive cells) and Hep3B (p53-negative cells), the authors showed that EGCG inhibited cell proliferation in a dose-dependent manner, thus suggesting that an additional factor to $\mathrm{p} 53$ is involved in promoting the inhibitory effect of EGCG on HCC proliferation. To demonstrate this, levels of AMPK, a sensor of cellular energy levels, was evaluated in both p53-positive and -negative human hepatoma cells. Data showed that EGCG was able to upregulate AMPK activity in HCC cells by inhibiting the mTOR pathway.

Moreover, by activation of AMPK, EGCG is able to decrease the activity and/or expression of lipogenic enzymes, such as ACC and FASN. All these findings support the hypothesis that EGCG could be potentially used as a chemopreventive and antilipogenic compound for HCC. Tong et $\mathrm{al}^{50}$ showed that the cytotoxic effect of EGCG on HepG2 cells was associated with activation of the TGF $\beta_{1}-$ Smad signaling pathway. Subsequently, the antitumor effect of EGCG on liver cancer was described in a murine model for chemoresistant HCC using doxorubicin (Dox) in combination with epicatechin gallate and EGCG. A synergistic effect between Dox and epicatechin gallate or EGCG administered together in vitro and in vivo was observed. Moreover, it has been proved that catechins enhance chemosensitivity to Dox, increase Dox cytotoxicity in HCC cells, and inhibit Pgp expression in Dox-resistant $\mathrm{HCC}$ cells in vitro and in xenograft tumors. An inhibitory effect of catechins on the expression of multidrug-resistance genes was likewise observed. ${ }^{51}$

A fascinating study supporting the chemopreventive effect of EGCG on HCC, was conducted in SMMC7721 and Hep3B cells. By in vitro and in vivo studies, the authors showed that EGCG was able to inhibit human CBR1 activity, which in turn converted the antitumor drug and anthracycline daunorubicin (Dnr) into the alcohol metabolite daunorubicinol, reducing antitumor activity and cardiotoxicity. Taken together, these data suggest that a combination of EGCG and Dnr might represent a novel approach for HCC chemoprevention. $^{52}$

Additional studies have confirmed the chemopreventive role of EGCG in HCC treatment. It has been shown that EGCG was able to sensitize HCC cells to 5-fluorouracil antitumor activity. Moreover, a synergistic effect between EGCG and 5-fluorouracil was detected in Hep3B cells, mainly through the downregulation of Cox 2 and hyperactivation of AMPK. ${ }^{53}$ An interesting in vitro study conducted on the HCC cell line HepG2, human hepatoma cell lines MHCC97L, MHCC97H, and the human hepatocyte cell line L02 showed that the anti-HCC effects of EGCG might be mediated by suppression of prostanoid EP1 expression and by the production of $\mathrm{PGE}_{2} \cdot{ }^{54}$ The anticancer effect of EGCG on HCC has also been proven on HCCLM6 cells. Using in vitro approaches, the authors demonstrated that EGCG arrested HCCLM6 cell metastasis by inhibiting MMP2 and MMP9 expression. Moreover, EGCG altered expression levels of FUBP1, HSPB1, CH60, and NPM proteins, strongly related to metastasis formation. ${ }^{41}$ Despite promising results on the antitumor effect of EGCG on $\mathrm{HCC}$, a report from Hashimoto et $\mathrm{al}^{55}$ described that a new methylated EGC analogue (methyl-EGCG) had a stronger antioxidation effect than EGCG in Huh7 hepatoma cells and corresponding xenografted hepatoma mice. A new molecular mechanism underlying the anticancer effect of EGCG on HCC cells was highlighted by Wang et al. ${ }^{56}$ By treating BEL7402 and QGY7703 cells with EGCG, they observed suppression of cell proliferation and a reduction in expression levels of phosphorylated STAT3 (p-STAT3), thus suggesting that regulation of STAT3 signaling could be considered as one of the molecular mechanisms underlying the anticancer effect of EGCG on HCC cells.

In another in vitro study, it has been shown that EGCG in HepG2, SMMC7721, and SK-Hep1 cells reduced the secretion of AFP, high levels of which are associated with malignant differentiation of cancer cells, through the modulation of autophagic activities of HCC cells. From these data, it emerged that EGCG inhibited HCC-cell growth by suppressing the Akt pathway. ${ }^{57}$

Different findings were depicted by Chen et al. ${ }^{46}$ The authors described a role of autophagy in the inhibitory effects 
on HCC cells of the synergistic interaction between EGCG and Dox. By using in vitro approaches on hepatoma cells and a subcutaneous Hep3B-cell xenograft tumor model, the authors showed that EGCG acts as a chemotherapeutic agent and acts synergistically by enhancing Dox anticancer effects through the inhibition of autophagy in HCC. The chemopreventive and hepatoprotective effects of EGCG against HCC were studied by Darweish et al in in vitro and Sprague Dawley rats. ${ }^{8}$ This investigation found that the heparan sulfate proteoglycans pathway was engaged with the chemopreventive and hepatoprotective effects of EGCG. In another study conducted on HCCLM6 cells and noncancerous liver cells (HL7702) treated with EGCG, it was shown that EGCG was able to induce apoptosis, decrease mitochondrial membrane potential, and promoted $\mathrm{G}_{0} / \mathrm{G}_{1}$-phase cell-cycle arrest only in HCCLM6 cells. ${ }^{58} \mathrm{An}$ interesting study was conducted on HepG2 and MHCC97H cells to investigate the role of EGCG in modulating OPN, which is a mediator of metastasis and invasion of HCC. Data reported showed that EGCG decreased OPN mRNA and secreted OPN protein levels by decreasing the half-life of OPN mRNA in MHCC97H cells..$^{59}$ An intriguing study was conducted on the effects of EGCG on human hepatoma and colon cancer stem cells, which are considered responsible for tumor recurrence and chemoresistance after hepatic resection. The authors showed that EGCG was able to inhibit the selfrenewal process in cancer stem cells of hepatoma and the colon by modulating the expression of ATP-binding cassettetransporter genes and stem-cell markers and by decreasing the transcription of NEK2 and $p$-Akt genes, thus resulting in Akt-signaling inhibition. ${ }^{60}$

An interesting investigation was conducted by $\mathrm{Li}$ et al ${ }^{61}$ on the inhibitory effects of EGCG on HCC-cell growth by involving the glycolysis process. In this study, it was reported that EGCG modulated the oligomeric structure of PFK, a metabolic sensor in the glycolytic pathway, and suppressed PFK activity. Moreover, EGCG enhanced the inhibitory effects of sorafenib on anaerobic HCC-cell growth and in an HCC-xenograft mouse model. Recently, it has been shown that EGCG attenuates the overexpression of Pgp induced by Dox in HepG2 cells by inhibiting the ERK-MAPK and PI3K-Akt signaling pathways. ${ }^{62}$ Finally, a new molecular mechanism underlying the EGCGmediated autophagic modulation of AFP in HepG2 cells has been proposed by Zhao et al. ${ }^{63}$ Taken together, all these findings suggest that EGCG could be conceivably utilized for counteracting and treating HCC by acting on different molecular mechanisms.

\section{Principal targets modulated by EGCG in hepatocellular cancer progression}

On the basis of the preclinical results described, it emerges that EGCG is able to regulate HCC progression by modulating principal molecular targets: $\mathrm{NF} \kappa \mathrm{B}$ and $\mathrm{p} 53$; ERK1/2 and PI3K-Akt-mTOR; and FGF and VEGF. As shown in Figure 1, the transition from HCC-tumor initiation to HCC proliferation is blocked by EGCG through downregulation of expression levels of p53, NFKB, EGFR, cyclins, and unfolded protein response and upregulation of BAX. Further, the switch from HCC proliferation to HCC invasion is inhibited by EGCG, mainly through the modulation of the expression levels of ERK1/2, PI3K-Akt-mTOR, STATs, multidrug resistance, AMPK, and caspases. Finally, EGCG greatly reduced $\mathrm{HCC}$ metastasis by downregulating expression of FGF, HIF, and MMPs and upregulating expression of IGF.

\section{In vivo investigations: animal models and EGCG effects}

The effects of EGCG on HCC have been additionally shown by in vivo experiments, as summarized in Table 2. In a fascinating experiment, Nishida et $\mathrm{al}^{36}$ reported that EGCG, given as $0.05 \%$ or $0.1 \%$ in drinking water for 7 weeks, reduced the incidence of spontaneous hepatocarcinogenesis in $\mathrm{C} 3 \mathrm{H} /$ HeNCrj mice. More recently, several investigations have been performed on ectopic xenograft murine models. In the aforementioned Nishikawa et al study ${ }^{40}$ the oral administration of EGCG $(0,0.8,2.5$, and $7.5 \mathrm{mg} / \mathrm{mL}$ ad libitum for 25 days) inhibited tumor growth and induced apoptosis in HLE xenograft tumors (HLE cells injected into the dorsal subcutaneous tissue of nude mice) by the downregulation of BCL2a and BCLxL in a dose-dependent manner. The authors also proved that high EGCG doses did not induce significant adverse effects on the growth or behavior of animals.

The efficacy of EGCG has been also investigated in vivo in combination with anticancer drugs. Liang et $\mathrm{a}^{51}$ evaluated the effect of EGC and EGCG on the antitumor activity of Dox in a murine xenograft model for chemoresistant HCC. In their experiments, the author found that the coadministration of Dox and low doses of EGCG significantly inhibited hepatoma growth. In addition, EGCG was able to increase the intracellular accumulation of Dox, due to the inhibition of Pgp and mRNA expression of several drug-resistance genes (eg, MDR1). Subsequently, the same authors proved that the synergistic effect of the combination of EGCG + Dox involved autophagy inhibition in a dose- and time-dependent manner. ${ }^{46}$ 


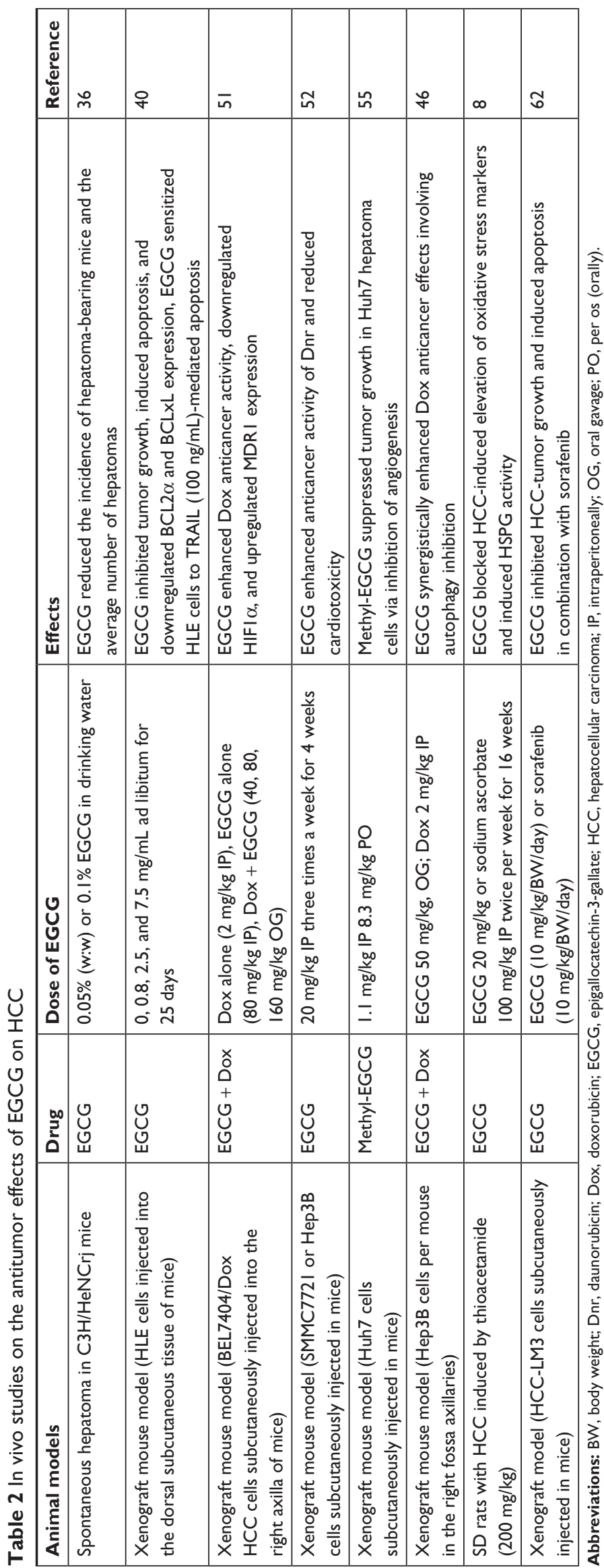


In another murine xenograft model obtained with SMMC7721 or Hep3B cells, EGCG enhanced the anticancer activity of the anthracycline Dnr through the inhibition of human CBR1, which is involved in its catabolism. ${ }^{52}$ Starting from the results of a previous experiment proving the biological activity of the methyl-EGCG analogue, Hashimoto et $\mathrm{al}^{55}$ demonstrated that oral administration of low-dose $(8.3 \mathrm{mg} / \mathrm{kg})$ methyl-EGCG inhibited cell growth in xenografted Huh7 hepatoma mice. In particular, methyl-EGCG was able to inhibit angiogenesis activity. EGCG-induced inhibition of vascular invasion was also studied by Darweish et al. ${ }^{8}$ In an animal model of HCC, the authors demonstrated that EGCG was able to induce restoration of HSPG receptors. In a murine xenograft model of $\mathrm{HCC}, \mathrm{Li}$ et $\mathrm{al}^{62}$ demonstrated that EGCG inhibited glycolysis and induced apoptosis in HCC cells through inhibition of the expression and activity of PFK, a limiting enzyme of glycolysis. As a consequence of this effect, EGCG improved the resistance of aerobic glycolytic HCC cells to sorafenib and enhanced cell-growth inhibition.

In the CAMARADES analysis (Table 3), we found that all the studies considered were published in peer-reviewed journals and showed detailed biochemical/tissue evaluations and professional statistical analysis. The newest studies ${ }^{8,62}$ obtained the highest score (9/11) despite the lack of any allocation-concealment technique, mandatory to prevent selection bias. This criterion is often underestimated, as demonstrated in our previous CAMARADES evaluations. ${ }^{64}$ Despite this gap, the results of all considered investigations were suggestive of a good-quality methodological approach in the research on these topics.

\section{Future research needs and translational perspectives in clinical practice}

Although experimental studies on the roles of EGCG in the prevention and treatment of HCC have demonstrated promising findings, further investigations are needed in order to understand the molecular basis underlying these effects. An intriguing issue concerns the mechanisms underlying cellcycle arrest and apoptosis in HCC. For instance, in contrast with results found in differentiated cell lines (eg, HepG2 cells), it has been reported that EGCG failed to induce cell-cycle arrest and had no effect on Fas expression in an undifferentiated cell line (HLE), whereas in these latter cells apoptosis was obtained via caspase 9 activation through inhibition of BCL2a and BCLxL. The translational point of view of these discoveries is exceptionally fascinating, as they suggest that in $\mathrm{HCC}-$ featuring rapid cell proliferation and strong expression of antiapoptotic genes - EGCG ought to work by affecting different apoptotic mechanisms in different phases of cancer development and progression. Additional investigations should be focused on better understanding the antiangiogenic effects of EGCG and the mechanisms involved in the activation of the immunoresponse against tumors. Positive responses in this field could stimulate preclinical investigation - and subsequently translational approaches in clinical practice based on the combination of EGCG with tumor-targeted and immunotargeted therapies in the treatment of HCC.

Liang et al' $\mathrm{s}^{51}$ results on the combination of EGCG and Dox could stimulate research on the synergy between EGCG and chemotherapy drugs on cell killing and potentially sensitizing

Table 3 Quality of evidence obtained using a modified CAMARADES checklist

\begin{tabular}{|c|c|c|c|c|c|c|c|c|}
\hline & \multicolumn{8}{|c|}{ References } \\
\hline & 36 & 40 & 51 & 46 & 52 & 55 & 8 & 62 \\
\hline Publication in a peer-reviewed journal & $\sqrt{ }$ & $\sqrt{ }$ & $\sqrt{ }$ & $\sqrt{ }$ & $\sqrt{ }$ & $\sqrt{ }$ & $\sqrt{ }$ & $\sqrt{ }$ \\
\hline Number of experiments and control-groups & $\sqrt{ }$ & $\sqrt{ }$ & $\sqrt{ }$ & $\sqrt{ }$ & & $\sqrt{ }$ & $\sqrt{ }$ & $\sqrt{ }$ \\
\hline Housing and husbandry conditions & $\sqrt{ }$ & $\sqrt{ }$ & & & & & $\sqrt{ }$ & $\sqrt{ }$ \\
\hline Details of intervention/exposure group procedures & $\sqrt{ }$ & $\sqrt{ }$ & $\sqrt{ }$ & $\sqrt{ }$ & & $\sqrt{ }$ & $\sqrt{ }$ & $\sqrt{ }$ \\
\hline Random allocation to groups & $\sqrt{ }$ & $\sqrt{ }$ & & $\sqrt{ }$ & & & & $\sqrt{ }$ \\
\hline \multicolumn{9}{|l|}{ Allocation concealment } \\
\hline Blinded assessment of outcome & & & $\sqrt{ }$ & & & & $\sqrt{ }$ & \\
\hline Biochemical/molecular evaluations & & $\sqrt{ }$ & $\sqrt{ }$ & $\sqrt{ }$ & $\sqrt{ }$ & $\sqrt{ }$ & $\sqrt{ }$ & $\sqrt{ }$ \\
\hline Tissue evaluations & $\sqrt{ }$ & $\sqrt{ }$ & $\sqrt{ }$ & $\sqrt{ }$ & $\sqrt{ }$ & $\sqrt{ }$ & $\sqrt{ }$ & $\sqrt{ }$ \\
\hline Statistical analysis & $\sqrt{ }$ & $\sqrt{ }$ & $\sqrt{ }$ & $\sqrt{ }$ & $\sqrt{ }$ & $\sqrt{ }$ & $\sqrt{ }$ & $\sqrt{ }$ \\
\hline Statement regarding possible conflict of interest & & & $\sqrt{ }$ & $\sqrt{ }$ & $\sqrt{ }$ & $\sqrt{ }$ & $\sqrt{ }$ & $\sqrt{ }$ \\
\hline Total (of II) & 7 & 8 & 8 & 8 & 5 & 7 & 9 & 9 \\
\hline
\end{tabular}

Note: $\sqrt{ }$, criterion satisfied. 
chemoresistant HCC cells to the latter. Furthermore, combinations of EGCG and anticancer drugs can represent a useful strategy to prevent and limit different side effects associated with antineoplastic drug therapy. ${ }^{65,66}$ In this regard, Huang et $\mathrm{al}^{52}$ showed us that EGCG could be tested in a clinical trial in order to prevent the cardiotoxicity of the anticancer drug Dnr.

Several clinical reports have indicated the use of greentea-based supplements as agents of liver toxicity. ${ }^{67}$ In addition, animal studies have indicated that EGCG-induced hepatotoxicity was the effect of increased IL6 activity combined with oxidative stress activation involving hepatic lipid peroxidation, plasma 8-isoprostane, and increased hepatic metallothionein and $\gamma$-histone $2 \mathrm{AX}$ protein expression. ${ }^{68}$ On the other hand, decreased superoxide dismutase and glutathione peroxidase levels suggest the potential role of mitochondriafunction inhibition due to high doses of EGCG. ${ }^{69}$ Probably, these alarming data restrain translational approaches. Preclinical research could offer suggestions to overcome these obstacles by the identification of the optimum therapeutic dosage of EGCG for intervention trials in patients suffering from $\mathrm{HCC}$ and by investigation into effective strategies to prevent EGCG-induced toxicity. For instance, Hashimoto et al's study indicated that modification of the 3-position by methylation in EGCG (methyl-EGCG) was effective in cell-growth inhibition at very low oral dosages. ${ }^{55}$ Finally, according to Garcia et al, ${ }^{70}$ we believe that a great limitation of the translation of preclinical data on EGCG and other flavonoids into clinical practice is the lack of standardized animal models.

\section{Conclusion}

Several preclinical studies reviewed here provide evidence that EGCG induces apoptosis and inhibits HCC progression by acting on different molecular pathways. These promising results encourage the use of EGCG in clinical practice. Despite the EGCG compound being currently used in clinical trials for the treatment of various type of cancer and other diseases, ${ }^{7}$ no clinical trials have been conducted for patients suffering from HCC. For these reasons, more studies are urgently needed. These studies should be focused on understanding molecular mechanisms regulated by EGCG in $\mathrm{HCC}$, identification of the optimum therapeutic dosage of EGCG to be used in clinical trials, and the identification of more effective strategies to prevent EGCG-induced toxicity.

\section{Acknowledgments}

We are grateful to Dr Alessandra Trocino and Mrs Cristina Romano from the National Cancer Institute of Naples for providing excellent bibliographic service and assistance.

\section{Author contributions}

This review was written mainly by SB and MC. RP and MC are co-last authors. All authors contributed toward data analysis, drafting and critically revising the paper, gave final approval of the version to be published, and agree to be accountable for all aspects of the work.

\section{Disclosure}

The authors report no conflicts of interest in this work.

\section{References}

1. Byam J, Renz J, Millis JM. Liver transplantation for hepatocellular carcinoma. Hepatobiliary Surg Nutr. 2013;2(1):22-30.

2. Center for Disease Control and Prevention (CDC) hepatocellular carcinoma - United States 2001-2006. MMWR Morb Mortal Wkly Rep. 2010;59(17):517-520.

3. Balogh J, Victor D, Asham EH, et al. Hepatocellular carcinoma: a review. J Hepatocell Carcinoma. 2016;3:41-53.

4. Niu L, Liu L, Yang S, Ren J, Lai PBS, Chen GG. New insights into sorafenib resistance in hepatocellular carcinoma: Responsible mechanisms and promising strategies. Biochim Biophys Acta. 1868;2017(2): 564-570.

5. Bruix J, Qin S, Merle P, et al. Regorafenib for patients with hepatocellular carcinoma who progressed on sorafenib treatment (RESORCE): a randomised, double-blind, placebo-controlled, phase 3 trial. Lancet. 2017;389(10064):56-66.

6. Cascella M, Bimonte S, Muzio MR, Schiavone V, Cuomo A. The efficacy of epigallocatechin-3-gallate (green tea) in the treatment of Alzheimer's disease: an overview of pre-clinical studies and translational perspectives in clinical practice. Infect Agent Cancer. 2017; 12(1):36.

7. Bimonte S, Leongito M, Barbieri A, et al. Inhibitory effect of (-)-epigallocatechin-3-gallate and bleomycin on human pancreatic cancer MiaPaca-2 cell growth. Infect Agent Cancer. 2015;10(1):22.

8. Darweish MM, Ebrahim MA, Al-Gayyar MM, Abbas A. Anticancer activity of EGCG against HCC. J Pharm Pharmacol. 2014;66: 1032-1045.

9. Zhang H, Cao D, Cui W, Ji M, Qian X, Zhong L. Molecular bases of thioredoxin and thioredoxin reductase-mediated prooxidant actions of (-)-epigallocatechin-3-gallate. Free Radic Biol Med. 2010;49(12): 2010-2018.

10. Zhou L, Elias RJ. Antioxidant and pro-oxidant activity of (-)-epigallocatechin-3-gallate in food emulsions: Influence of $\mathrm{pH}$ and phenolic concentration. Food Chem. 2013;138(2-3):1503-1509.

11. Farhan M, Rizvi A, Naseem I, Hadi SM, Ahmad A. Targeting increased copper levels in diethylnitrosamine induced hepatocellular carcinoma cells in rats by epigallocatechin-3-gallate. Tumor Biol. 2015;36(11): 8861-8867.

12. Farhan M, Khan HY, Oves M, et al. Cancer therapy by catechins involves redox cycling of copper ions and generation of reactive oxygen species. Toxins. 2016;8(2):37.

13. Macleod MR, O'Collins T, Howells DW, Donnan GA. Stroke pooling of animal experimental data reveals influence of study design and publication bias. 2004;35:1203-1208.

14. Bimonte S, Cascella M, Schiavone V, Mehrabi-Kermani F, Cuomo A. The roles of epigallocatechin-3-gallate in the treatment of neuropathic pain: an update on preclinical in vivo studies and future perspectives. Drug Des Devel Ther. 2017;11:2737-2742.

15. Khan HY, Zubair H, Ullah MF, Ahmad A, Hadi SM. Oral administration of copper to rats leads to increased lymphocyte cellular DNA degradation by dietary polyphenols: implications for a cancer preventive mechanism. Biometals. 2011;24(6):1169-1178. 
16. Gupte A, Mumper RJ. Elevated copper and oxidative stress in cancer cells as a target for cancer treatment. Cancer Treat Rev. 2009;35(1): $32-46$.

17. Lambert JD, Lee MJ, Lu H, et al. Epigallocatechin-3-gallate is absorbed but extensively glucuronidated following oral administration to mice. J Nutr. 2003;133(12):4172-4177.

18. Lu H, Meng X, Yang CS. Enzymology of methylation of tea catechins and inhibition of catechol-O-methyltransferase by (-)-epigallocatechin gallate. Drug Metab Dispos. 2003;31(5):572-579.

19. Liu AB, Tao S, Lee MJ, et al. Effects of gut microbiota and time of treatment on tissue levels of green tea polyphenols in mice. BioFactors. 2018;44(4):348-360.

20. Chen C, Liu Q, Liu L, Hu YY, Feng Q. Potential biological effects of (-)-epigallocatechin-3-gallate on the treatment of nonalcoholic fatty liver disease. Mol Nutr Food Res. 2018;62(1):1700483.

21. Lee MJ, Maliakal P, Chen L. Pharmacokinetics of tea catechins after ingestion of green tea and (-)-epigallocatechin-3-gallate by humans: formation of different metabolites and individual variability. Cancer Epidemiol Biomark Prev. 2002;11:1025-1032.

22. Altland K, Schreiner R, Hunstein W. Of green tea, black pepper, and amyloidoses; 2010. Available from: http://www.ukgm.de/ugm_2/deu/ ugi_hum/EGCG_Piperin_engl.pdf. Accessed July 13, 2011.

23. Auger C, Mullen W, Hara Y, Crozier A. Bioavailability of polyphenon E flavan-3-ols in humans with an ileostomy. J Nutr. 2008;138(8): $1535 \mathrm{~S}-1542 \mathrm{~S}$

24. Stalmach A, Troufflard S, Serafini M, Crozier A. Absorption, metabolism and excretion of Choladi green tea flavan-3-ols by humans. Mol Nutr Food Res. 2009;53(Suppl 1):S44-S53.

25. Roowi S, Stalmach A, Mullen W, Lean ME, Edwards CA, Crozier A. Green tea flavan-3-ols: colonic degradation and urinary excretion of catabolites by humans. J Agric Food Chem. 2010;58(2):1296-1304.

26. Mereles D, Hunstein W. Epigallocatechin-3-gallate (EGCG) for clinical trials: more pitfalls than promises? Int J Mol Sci. 2011;12(9):5592-5603.

27. Li N, Taylor LS, Mauer LJ. Degradation kinetics of catechins in green tea powder: effects of temperature and relative humidity. J Agric Food Chem. 2011;59(11):6082-6090.

28. Ishii T, Ichikawa $\mathrm{T}$, Minoda $\mathrm{K}$, et al. Human serum albumin as an antioxidant in the oxidation of (-)-epigallocatechin gallate: participation of reversible covalent binding for interaction and stabilization. Biosci Biotechnol Biochem. 2011;75(1):100-106.

29. Hunstein W. AL-amyloidosis and epigallocatechin-3-gallate: 4 years and 5 months later; 2011. Available from: http://www.hunstein-egcg.de. Accessed July 13, 2011.

30. Peters CM, Green RJ, Janle EM, Ferruzzi MG. Formulation with ascorbic acid and sucrose modulates catechin bioavailability from green tea. Food Res Int. 2010;43(1):95-102.

31. Giunta B, Hou H, Zhu Y, et al. Fish oil enhances anti-amyloidogenic properties of green tea EGCG in Tg2576 mice. Neurosci Lett. 2010; 471(3):134-138.

32. Dube A, Nicolazzo JA, Larson I. Chitosan nanoparticles enhance the intestinal absorption of the green tea catechins (+)-catechin and (-)-epigallocatechin gallate. Eur J Pharm Sci. 2010;41(2):219-225.

33. Hu B, Ting Y, Yang X, Tang W, Zeng X, Huang Q. Nanochemoprevention by encapsulation of (-)-epigallocatechin-3-gallate with bioactive peptides/chitosan nanoparticles for enhancement of its bioavailability. Chem Commun. 2012;48(18):2421-2423.

34. Vyas S, Sharma M, Sharma PD, Singh TV, Design STV. Design, semisynthesis, and evaluation of $O$-acyl derivatives of (-)-epigallocatechin3-gallate as antitumor agents. J Agric Food Chem. 2007;55(15): 6319-6324.

35. Tanaka H, Miyoshi H, Chuang YC, Ando Y, Takahashi T. Solid-phase synthesis of epigallocatechin gallate derivatives. Angew Chem Int Ed. 2007;46(31):5934-5937.

36. Nishida H, Omori M, Fukutomi Y, et al. Inhibitory effects of (-)-epigallocatechin gallate on spontaneous hepatoma in $\mathrm{C} 3 \mathrm{H} / \mathrm{HeNCrj}$ mice and human hepatoma-derived PLC/PRF/5 cells. Jpn J Cancer Res. 1994;85(3):221-225.
37. Zhang G, Miura Y, Yagasaki K. Suppression of adhesion and invasion of hepatoma cells in culture by tea compounds through antioxidative activity. Cancer Lett. 2000;159(2):169-173.

38. Uesato S, Kitagawa Y, Kamishimoto M, Kumagai A, Hori H, Nagasawa $\mathrm{H}$. Inhibition of green tea catechins against the growth of cancerous human colon and hepatic epithelial cells. Cancer Lett. 2001; 170(1):41-44.

39. Park HJ, Shin DH, Chung WJ, et al. Epigallocatechin gallate reduces hypoxia-induced apoptosis in human hepatoma cells. Life Sci. 2006; 78(24):2826-2832.

40. Nishikawa T, Nakajima T, Moriguchi M, et al. A green tea polyphenol, epigalocatechin-3-gallate, induces apoptosis of human hepatocellular carcinoma, possibly through inhibition of BCL2 family proteins. J Hepatol. 2006;44(6):1074-1082.

41. Zhang Y, Owusu L, Duan W, et al. Anti-metastatic and differential effects on protein expression of epigallocatechin-3-gallate in HCCLM6 hepatocellular carcinoma cells. Int J Mol Med. 2013;32(4):959-964.

42. Zhang Q, Tang X, Lu Q, Zhang Z, Rao J, Le AD. Green tea extract and (-)-epigallocatechin-3-gallate inhibit hypoxia- and serum-induced HIF1alpha protein accumulation and VEGF expression in human cervical carcinoma and hepatoma cells. Mol Cancer Ther. 2006;5(5): $1227-1238$.

43. Lee SJ, Lee KW, Hur HJ, Chun JY, Kim SY, Lee HJ. Phenolic phytochemicals derived from red pine (Pinus densiflora) inhibit the invasion and migration of SK-HEP-1 human hepatocellular carcinoma cells. Ann N Y Acad Sci. 2007;1095(1):536-544.

44. Lee SI, Kim HJ, Boo YC. Effect of green tea and (-)-epigallocatechin gallate on ethanol-induced toxicity in HepG2 cells. Phytother Res. 2008; 22(5):669-674.

45. Shimizu M, Shirakami Y, Sakai H, et al. EGCG inhibits activation of the insulin-like growth factor (IGF)/IGF1 receptor axis in human hepatocellular carcinoma cells. Cancer Lett. 2008;262(1):10-18.

46. Chen L, Ye HL, Zhang G, et al. Autophagy inhibition contributes to the synergistic interaction between EGCG and doxorubicin to kill the hepatoma Hep3B cells. PLoS One. 2014;9(1):e85771.

47. Kaufmann R, Henklein P, Henklein P, Settmacher U. Green tea polyphenol epigallocatechin-3-gallate inhibits thrombin-induced hepatocellular carcinoma cell invasion and p42/p44-MAPKinase activation. Oncol Rep. 2009;21(5):1261-1267.

48. Shirakami Y, Shimizu M, Adachi S, et al. (-)-Epigallocatechin gallate suppresses the growth of human hepatocellular carcinoma cells by inhibiting activation of the vascular endothelial growth factor-vascular endothelial growth factor receptor axis. Cancer Sci. 2009;100(10): 1957-1962.

49. Huang CH, Tsai SJ, Wang YJ, Pan MH, Kao JY, Way TD. EGCG inhibits protein synthesis, lipogenesis, and cell cycle progression through activation of AMPK in $\mathrm{p} 53$ positive and negative human hepatoma cells. Mol Nutr Food Res. 2009;53(9):1156-1165.

50. Tong JL, Nie F, Ran ZH, et al. [Epigallocatechin gallate induces apoptosis in human hepatocellular carcinoma HepG2 cells via TGF/Smad signaling pathway]. Zhonghua Zhong Liu Za Zhi. 2009;31(9):646-650. Chinese.

51. Liang G, Tang A, Lin X, et al. Green tea catechins augment the antitumor activity of doxorubicin in an in vivo mouse model for chemoresistant liver cancer. Int J Oncol. 2010;37(1):111-123.

52. Huang W, Ding L, Huang Q, et al. Carbonyl reductase 1 as a novel target of (-)-epigallocatechin gallate against hepatocellular carcinoma. Hepatology. 2010;52(2):703-714.

53. Yang XW, Wang XL, Cao LQ, et al. Green tea polyphenol epigallocatechin3-gallate enhances 5-fluorouracil-induced cell growth inhibition of hepatocellular carcinoma cells. Hepatol Res. 2012;42(5):494-501.

54. Jin J, Chang Y, Wei W, et al. Prostanoid EP1 receptor as the target of (-)-epigallocatechin-3-gallate in suppressing hepatocellular carcinoma cells in vitro. Acta Pharmacologica Sinica. 2012;33(5):701-709.

55. Hashimoto O, Nakamura A, Nakamura T, et al. Methylated-(3")epigallocatechin gallate analog suppresses tumor growth in Huh7 hepatoma cells via inhibition of angiogenesis. Nutr Cancer. 2014;66(4):728-735. 
56. Wang Y, Ren X, Deng C, et al. Mechanism of the inhibition of the STAT3 signaling pathway by EGCG. Oncol Rep. 2013;30(6):2691-2696.

57. Shen X, Zhang Y, Feng Y, et al. Epigallocatechin-3-gallate inhibits cell growth, induces apoptosis and causes $\mathrm{S}$ phase arrest in hepatocellular carcinoma by suppressing the Akt pathway. Int J Oncol. 2014;44(3): 791-796.

58. Zhang Y, Duan W, Owusu L, Wu D, Xin Y. Epigallocatechin-3-gallate induces the apoptosis of hepatocellular carcinoma LM6 cells but not non-cancerous liver cells. Int J Mol Med. 2015;35(1):117-124.

59. Zapf MA, Kothari AN, Weber CE, et al. Green tea component epigallocatechin-3-gallate decreases expression of osteopontin via a decrease in mRNA half-life in cell lines of metastatic hepatocellular carcinoma Surgery. 2015;158(4):1039-1048.

60. Wubetu GY, Shimada M, Morine Y, et al. Epigallocatechin gallate hinders human hepatoma and colon cancer sphere formation. J Gastroenterol Hepatol. 2016;31(1):256-264.

61. Li S, Wu L, Feng J, et al. In vitro and in vivo study of epigallocatechin3-gallate-induced apoptosis in aerobic glycolytic hepatocellular carcinoma cells involving inhibition of phosphofructokinase activity. Sci Rep. 2016;6(1):28479.

62. Satonaka H, Ishida K, Takai M, et al. (-)-Epigallocatechin-3-gallate Down-regulates Doxorubicin-induced Overexpression of P-glycoprotein Through the Coordinate Inhibition of PI3K/Akt and Mek/ERK Signaling Pathways. Anticancer Res. 2017;37(11):6071-6077.
63. Zhao L, Liu S, Xu J, et al. A new molecular mechanism underlying the EGCG-mediated autophagic modulation of AFP in HepG2 cells. Cell Death Dis. 2017;8(11):e3160.

64. Cascella M, Palma G, Barbieri A, et al. Role of Nigella sativa and its constituent thymoquinone on chemotherapy-induced nephrotoxicity: Evidences from experimental animal studies. Nutrients. 2017;9(6):625.

65. Cascella M. Chemotherapy-induced peripheral neuropathy: limitations in current prophylactic strategies and directions for future research. Curr Med Res Opin. 2017;33(6):981-984.

66. Cascella M. Preoperative cardiac evaluation and anesthetic considerations for cancer patients who underwent chemotherapy. Trends Anaesth Crit Care. 2017;14:9-18.

67. Jimenez-Saenz M, Martinez-Sanchez MC. Acute hepatitis associated with the use of green tea infusions. J Hepatol. 2006;44(3):616-617.

68. Lambert JD, Kennett MJ, Sang S, Reuhl KR, Ju J, Yang CS Hepatotoxicity of high oral dose (-)-epigallocatechin-3-gallate in mice. Food Chem Toxicol. 2010;48(1):409-416.

69. James KD, Kennett MJ, Lambert JD. Potential role of the mitochondria as a target for the hepatotoxic effects of (-)-epigallocatechin-3-gallate in mice. Food Chem Toxicol. 2018;111:302-309.

70. García ER, Gutierrez EA, Melo Fcsade, Novaes RD, Gonçalves RV. Flavonoids effects on hepatocellular carcinoma in murine models: a systematic review. Evid Based Complement Alternat Med. 2018;2018: 6328970 .
Drug Design, Development and Therapy

\section{Publish your work in this journal}

Drug Design, Development and Therapy is an international, peerreviewed open-access journal that spans the spectrum of drug design and development through to clinical applications. Clinical outcomes, patient safety, and programs for the development and effective, safe, and sustained use of medicines are the features of the journal, which

\section{Dovepress}

has also been accepted for indexing on PubMed Central. The manuscript management system is completely online and includes a very quick and fair peer-review system, which is all easy to use. Visit http://www.dovepress.com/testimonials.php to read real quotes from published authors.

Submit your manuscript here: http://www.dovepress.com/drug-design-development-and-therapy-journal 